\title{
Satellite differential SAR interferometry for the evaluation of effects of hydrogeological disasters: definition of a scale for damage evaluation
}

\author{
Vincenzo Rizzo $\left({ }^{1}\right)$ and Antonio Iodice $\left(^{2}\right)$ \\ (1) Istituto di Ricerca per la Protezione Idrogeologica (IRPI), CNR, Rende (CS), Italy \\ $\left.{ }^{2}\right)$ Dipartimento di Ingegneria Elettronica e delle Telecomunicazioni, \\ Università degli Studi di Napoli «Federico II», Napoli, Italy
}

\begin{abstract}
In this paper we present the results of monitoring soil movements over an about $10 \mathrm{~km}^{2}$ area around the border between the Calabria and Basilicata regions in Italy. Monitoring has been performed using the satellite differential SAR interferometry measurements integrated with GPS measurements. In particular, we used ERS data acquired at time interval of several months (about two acquisitions per year), and after particularly strong pluvial events. Terrain displacement spatial and temporal analysis has been performed by employing the original method described in Berardino et al. $(2002,2003)$. Obtained results allow us to characterise unstable areas, and can be used within projects aimed at territory classification and characterisation, and at performing damage evaluation. Accordingly, this technique has been included in the framework of a proposed UE project (WEDELOP project) aimed at developing an integrated methodology to devise a damage scale for hydrogeological disasters. This scale is of great interest in many fields; in particular, definition of a damage scale would be highly desirable from the viewpoint of insurance companies.
\end{abstract}

Key words landslide movements - damage evaluation - differential SAR interferometry - remote sensing of land surface - Maratea

\section{Rationale and methods}

Natural disasters caused by meteorological phenomena (such as hail, storms, floods, landslides), are affecting countries of the European Union with increasing frequency, and are producing heavier and heavier damage, partly hampering the economic development of these countries.

Mailing address: Dr. Antonio Iodice, Dipartimento di Ingegneria Elettronica e delle Telecomunicazioni, Università degli Studi di Napoli «Federico II», Via Claudio 21, 80125 Napoli, Italy; e-mail: iodice@unina.it
Timely assessment of damage caused by natural disasters and, above all, their continuous monitoring, are of fundamental importance in a modern well organised society to understand the effects of climatic changes on the social and economic fabric. In fact, a fast and synoptic data acquisition is useful to guarantee rapid adequate intervention, and, on the other hand, it is fundamental in planning proper long-period countermeasures. In addition, loss pricing is highly interesting for institutions for socio-economic analysis, and also for insurance companies. Most of such institutions and companies produce their analysis or set their annual premium on the basis of estimates of damage in the last period. Currently, these estimates are based on very rough damage assessment performed immediately after the disaster by central or local administrations of the various European coun- 
tries. This assessment is largely approximated, and usually direct and indirect costs for damage fixing and for productive activity re-starting tend to significantly increase with respect to the initial estimate, due to necessarily unpredictable factors. In general, objective and uniform criteria for damage evaluation are still lacking, also in the case of low intensity, but more frequent, events. Direct on site evaluation of damaged objects is sometimes impossible, and is often very costly. The consequence of such a situation is that large-scale reliable estimates are not available.

By using modern well-established satellite remote sensing techniques, integrated with on site studies and measurements, an ad-hoc research can be conducted, aimed at defining damage scales. They will be functions of the event typology, its intensity, the urbanization degree, and so on. In order to do that, researches are needed on «sample events» used as a reference and to be investigated in detail.

Damaging events can be defined and coded first of all in terms of physical intensity using remote sensed data related to the considered event (e.g., height or duration of rain, wind strength, etc.). To these elements, some others can be added, relative to the intensity of effects on the physical environment. In fact, nowadays sufficiently experimented tools and methods are available for the analysis of soil changes and for the evaluation of the physical intensity of events. Physical effects at the soil produced by strong meteorological events consist of changes that are partly temporary (such as soil saturation, flooding, sea strength, etc.) and partly permanent (such as debris cover, terrain displacements, variation of coastline, etc.). Such effects can be detected and measured using remote sensing techniques (spectral and multispectral sensors, differential SAR interferometry, etc.), and then coded in terms of their intensity. The coding of the intensity of physical effects measured on the territory (for instance in terms of percentage of affected areas or number of damaged sites per investigated area) will be then related to the coding of the event intensity. In order to obtain a better homogeneity of data, it is useful to consider a first distinction among different homogeneous physical and morphological environments (Alpi- ne or Appenninic areas, coastal area, basins extension, kinds of rocks, etc.).

Accordingly, a number of possibly recent and well documented «sample events» that correspond to the above cited coding will be chosen. This set of events will be the basis for a detailed damage estimate, limited to areas that are representative of different urbanization degrees. Obtained data will allow the availability of a large survey to establish a scale of relations among events, physical effects produced, and damage that allows an estimate relying on objective coded criteria.

This general procedure was recently suggested within the 6th Framework Programme («Weather Damage Evaluation and Loss Pricing», or WEDELOP proposal) for a large number of weather risks. In particular with regard to landslide activity, permanent effects should be monitored in terms of slope movements, and a specific methodology could be assessed using remote sensing data.
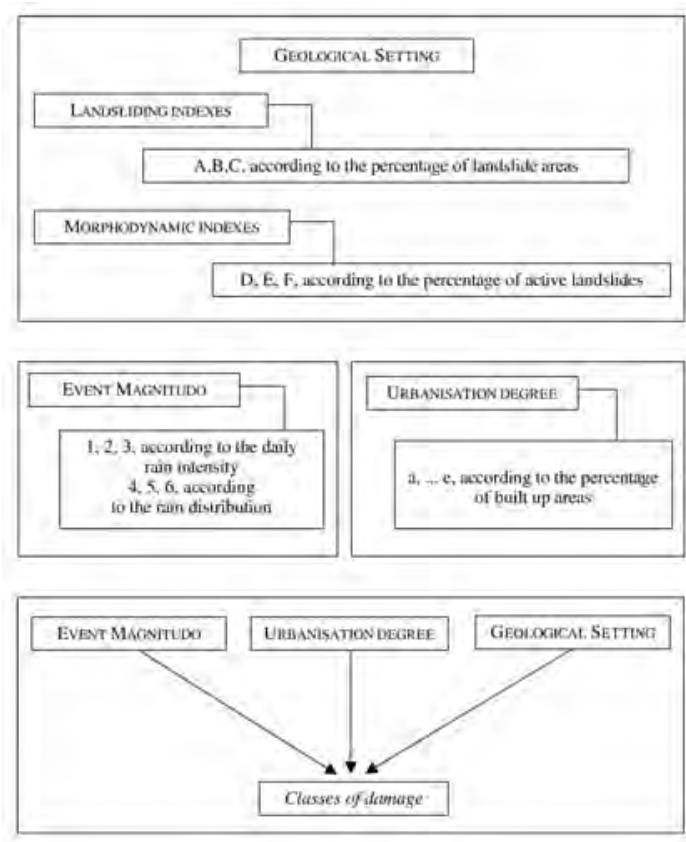

Fig. 1. Block scheme for the damage scale definition procedure. 


\section{Application to landslide damage evaluation}

Following the procedures proposed in WEDELOP we could also define a scale of damage for landslides scattered by rainy events whose elements are shown in fig. 1. The combination of these elements produces a large number of damage situations (and sampled events) to price: each combination of values of the geological settings, event magnitude, and urbanization degree indexes leads to one class of damage. However, to assess damage on large areas, some simplification can be made because only some factors are dominant. For example with regard to geological setting the «landsliding» and «morphodynamic» indexes are generally related: geodynamic areas showing a considerable amount of landslide are usually also characterized by a percentage increase in active landslide. More properly in certain cases (where available) the indexes of morphodynamic activity could be represented by the percentage of landslide remobilisation scattered by

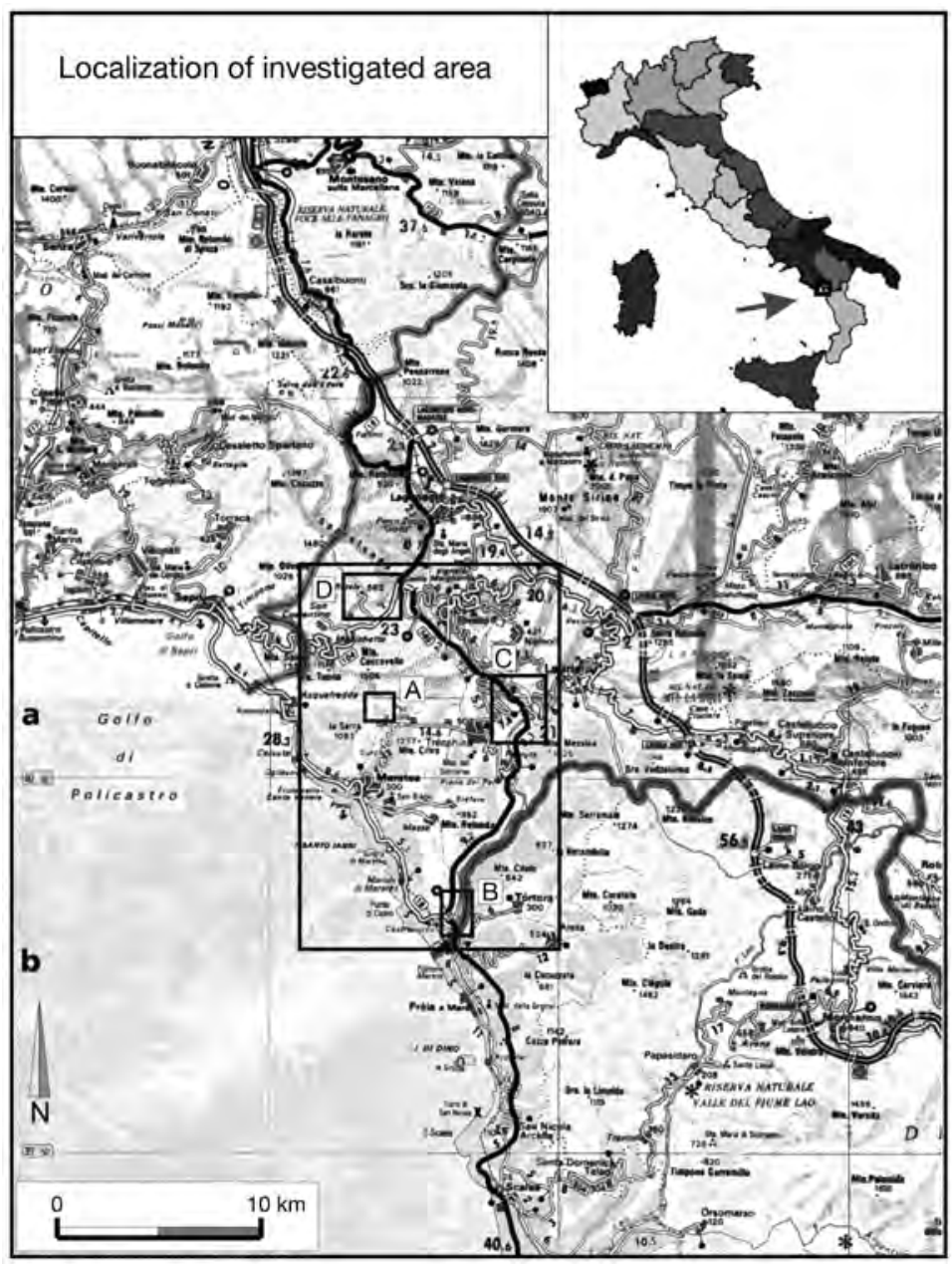

Fig. 2. The investigated area. Boxes A, B, C, and D show the locations of areas in the maps of fig. 5 . 
destabilising events. Finally, the degree of urbanisation is very important in relation to the potential damage, and should be immediately related to the events magnitude.

Geological setting (landslide and morphodynamic indexes for various lithological complexes) could be fixed on sampling areas; while differential SAR interferometry could help in assessing and setting up landslide activity, so that the morphodinamic index $(\mathrm{D}, \mathrm{E}, \mathrm{F})$ can be updated.

\section{Results of DiffSAR application on Earth slope movements performed at Maratea}

Differential Synthetic Aperture Radar (SAR) interferometry (DiffSAR) is able, in principle, to measure very small movements of the ground and to cover in continuity large areas (Godstein et al., 1994; Franceschetti and Lanari, 1999; Ferretti et al., 2001), so that it can be considered an ideal tool to investigate landslides and other slope instability (Acache et al., 1995; Carnec et al., 1996). In our implementation of the DiffSAR technique, the problem of decorrelation noise is faced using a phase unwrapping approach that allows us to process sparse data, and the impact of atmospheric artefacts is reduced by performing a temporal analysis of the deformations observed in successive interferograms.

As an example of the potentiality of this technique, we here show some results obtained by using it to improve our knowledge of the slope instability of a well investigated area (the Maratea Valley, see fig. 2) affected by continuous slow movements (Berardino et al., 2002, 2003).

In particular, using this technique, and employing the SAR acquisitions reported in table I, we analysed the time evolution of ground movements from 1997 to 2000 . This time interval had also been explored in the past using other techniques, such as distancemeter (EDM) and GPS measurements: GPS and DiffSAR results were then compared.

Obtained results are summarised in figs. 3 to 5. In the map of fig. 3, by visual inspection it is possible to verify the agreement between data obtained by the SAR technique (represented by the color scale defined in the bottom left of fig. 3) and those obtained by in situ GPS measurements (blue arrows). A 3D view of the map is reported in fig. 4, and a table of SAR versus GPS results is shown in table II. A full discussion of the comparison between SAR and GPS measurements can be found in (Berardino et al., 2003). We here want to emphasize that the employed technique is also able to detect small scale events, possibly with antropic origin, as shown in fig. 5.

Results of fig. 3 have shown that it is possible to perform a temporal analysis of continuous slow landslide movements using a limited number of ERS SAR data sets and low precision topographic information. All the acquired data (EDM, GPS and DiffSAR) are consistent and allow a kinematic model of instability within the investigated time interval to be sketched. A map

Table I. Interferometric pairs combined to generate the map of fig. 3.

\begin{tabular}{lcccc}
\hline \hline \multicolumn{2}{c}{ Dates of SAR acquisitions } & $\begin{array}{c}\text { Spatial baseline } \\
B_{\perp}[\mathrm{m}]\end{array}$ & $\begin{array}{c}\text { Temporal baseline } \\
\text { [days] }\end{array}$ & $\begin{array}{c}\text { Percentage of selected points } \\
\text { (coherence }>0.3)\end{array}$ \\
\hline $13 / 08 / 1997$ & $31 / 12 / 1997$ & 30 & 140 & $33.11 \%$ \\
$31 / 12 / 1997$ & $15 / 04 / 1998$ & 15 & 105 & $46.74 \%$ \\
$13 / 08 / 1997$ & $15 / 04 / 1998$ & 45 & 245 & $30.34 \%$ \\
$15 / 04 / 1998$ & $02 / 09 / 1998$ & 110 & 140 & $30.85 \%$ \\
$02 / 09 / 1998$ & $09 / 06 / 1999$ & 2 & 280 & $33.17 \%$ \\
$02 / 09 / 1998$ & $14 / 07 / 1999$ & 6 & 315 & $35.28 \%$ \\
$09 / 06 / 1999$ & $14 / 07 / 1999$ & 4 & 35 & $52.46 \%$ \\
$09 / 06 / 1999$ & $05 / 01 / 2000$ & 6 & 210 & $32.05 \%$ \\
$14 / 07 / 1999$ & $05 / 01 / 2000$ & 10 & 175 & $29.53 \%$ \\
\hline
\end{tabular}




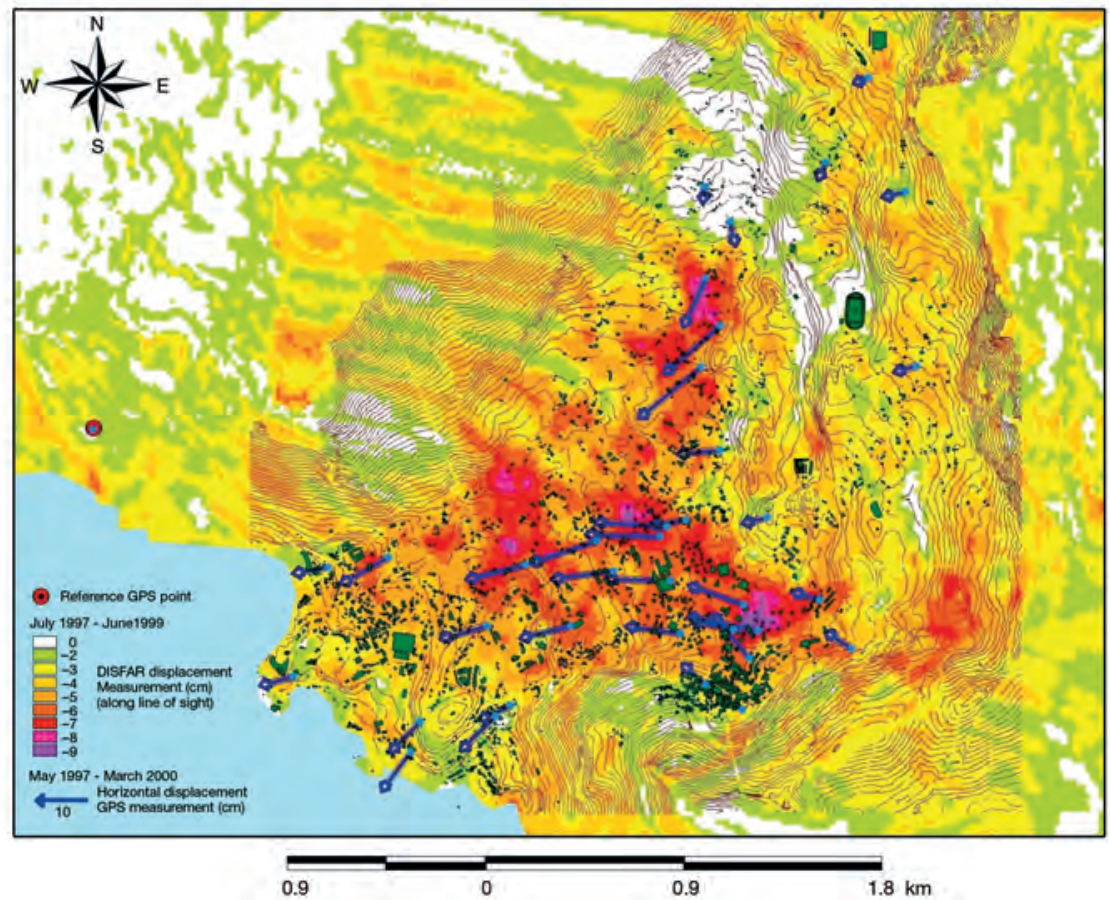

Fig. 3. DiffSAR displacement map relative to the period from July 1997 to January 2000, and GPS data acquired in almost the same time interval. Displacements must be understood as projected along the SAR line of sight (about $23^{\circ}$ from vertical and $104^{\circ}$ from north direction, descending orbit).

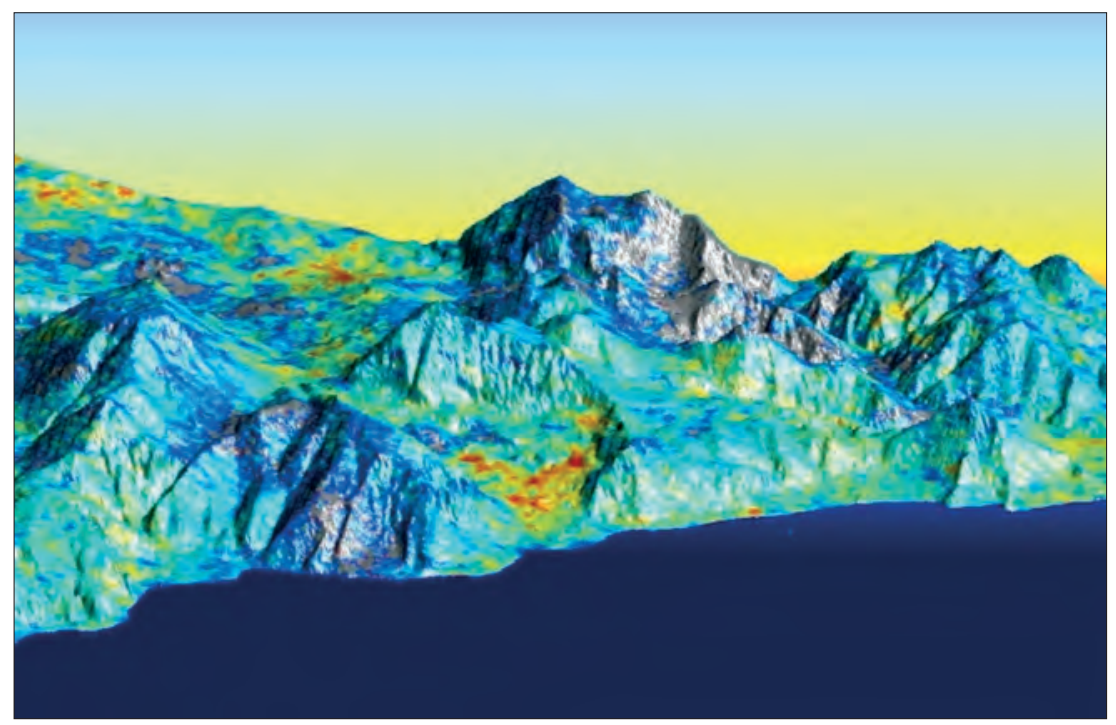

Fig. 4. DEM of the investigated area, showing DiffSAR displacements. The same color scale of fig. 3 is used, except for blue areas, showing slight positive vertical displacements: those are often low coherence area. 

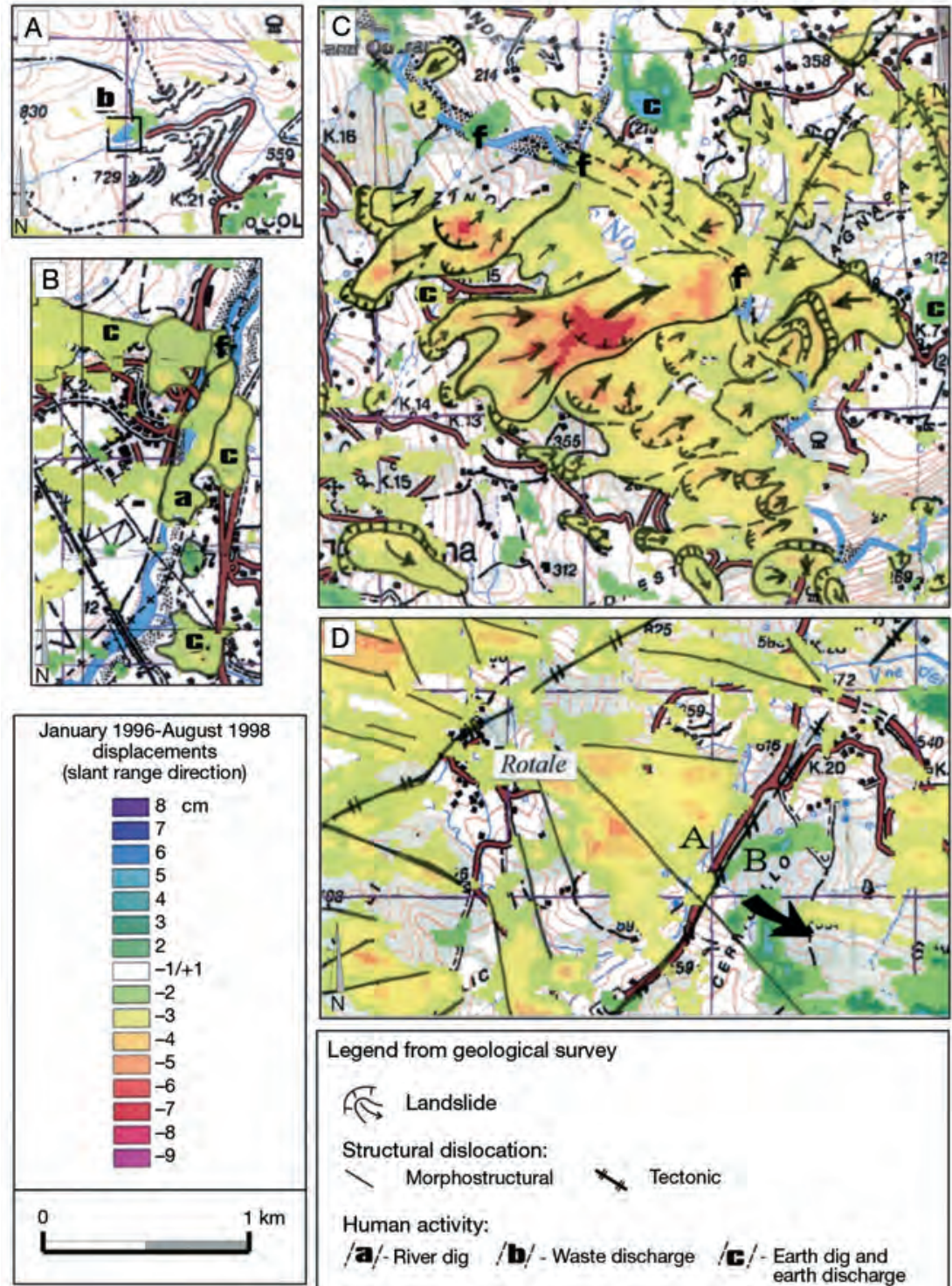

$$
\begin{aligned}
& \text { Legend from geological survey } \\
& \text { Structural dislocation: } \\
& \text { Morphostructural } \\
& / \mathbf{a} / \text { - River dig } / \mathbf{b} / \text { - Waste discharge /E / / - Earth dig and } \\
& \text { earth discharge } \\
& \text { /f/-Fluvial erosion }
\end{aligned}
$$

Fig. 5. Examples of geological validation for small phenomena, also of antropic nature, in the Maratea area (parts of maps, in scale 1:25000, of the vertical soil movements in the interval 1996-1998, only for pixels with coherence coefficient greater than 0.3). Top left (A): the Maratea dump (a few pixels large) is perfectly visible and well delimited, and turns out to be moving towards the sensor (i.e., upwards). Bottom left (B): last part of the Noce River, where detected upward and downward movements are coherent with erosion and deposition phenomena, with dumps, quarries, and so on. Top right (C): central part of the Noce Valley, where detected upward and downward movements are in reasonable agreement with low and progressive landslide movements. Bottom right (D): movements to be verified by GPS measurements, probably related to deep gravitative movements or tectonic alignments. 
Table II. Comparison of GPS and DiffSAR measurements. Displacements are both relative to the period from June 1997 to March 2000 and are projected along the SAR line of sight. The obtained values show good agreement in the pixels at higher coherence. However, by considering all the points, the difference between GPS and DiffSAR measurements has a mean value equal to $2.3 \mathrm{~cm}$ (DiffSAR underestimates the movements) and a standard deviation equal to $3.3 \mathrm{~cm}$. These discrepancies can be explained by considering that in the low-coherence points the DiffSAR measurements are obtained by interpolating results obtained in surrounding high-coherence points, so that they can be considered as average displacement over a rather wide area, whereas GPS measurements are strictly referred to each single ground point.

\begin{tabular}{|c|c|c|c|c|}
\hline $\begin{array}{c}\text { GPS } \\
\text { benchmarck } \\
\text { number }\end{array}$ & $\begin{array}{c}\text { GPS } \\
\text { displacement } \\
(\mathrm{mm})\end{array}$ & $\begin{array}{c}\text { DiffSAR } \\
\text { displacement } \\
(\mathrm{mm})\end{array}$ & Coherence & $\begin{array}{l}\text { GPS-SAR } \\
\text { difference } \\
(\mathrm{mm})\end{array}$ \\
\hline 1 & -52 & -54 & 0.3 & 2 \\
\hline 2 & -49 & -63 & 0.6 & 14 \\
\hline 3 & -65 & -63 & 0.5 & -2 \\
\hline 4 & -52 & -54 & 0.7 & 2 \\
\hline 5 & -69 & -22 & 0.2 & -47 \\
\hline 6 & -75 & -24 & 0.6 & -51 \\
\hline 7 & -18 & -15 & 0.2 & -3 \\
\hline 8 & -51 & -8 & 0.3 & -43 \\
\hline 9 & no & -25 & 0.6 & - \\
\hline 10 & -28 & -6 & 0.2 & -22 \\
\hline 11 & -84 & -56 & 0.2 & -28 \\
\hline 12 & -13 & -36 & 0.2 & 23 \\
\hline 13 & -105 & -83 & 0.7 & -22 \\
\hline 14 & -73 & -52 & 0.3 & -21 \\
\hline 15 & no & -47 & 0.3 & - \\
\hline 16 & -32 & -65 & 0.3 & 33 \\
\hline 17 & -17 & -38 & 0.7 & 21 \\
\hline 18 & -112 & -46 & 0.6 & -66 \\
\hline 19 & no & -14 & 0.4 & - \\
\hline 20 & -41 & -15 & 0.4 & -26 \\
\hline 21 & -102 & -27 & 0.6 & -80 \\
\hline 22 & no & -58 & 0.3 & - \\
\hline 23 & -97 & -51 & 0.3 & -46 \\
\hline 24 & no & -69 & 0.3 & - \\
\hline 25 & no & -69 & 0.3 & - \\
\hline 26 & -104 & -56 & 0.6 & -48 \\
\hline 27 & -24 & -75 & 0.2 & 51 \\
\hline 28 & -31 & -18 & 0.3 & -13 \\
\hline 29 & no & 2 & 0.2 & - \\
\hline 30 & -47 & -41 & 0.3 & -6 \\
\hline 31 & -24 & -18 & 0.2 & -6 \\
\hline 32 & -120 & -33 & 0.1 & -87 \\
\hline 33 & -97 & -13 & 0.2 & -84 \\
\hline 34 & -83 & -68 & 0.4 & -15 \\
\hline 35 & -66 & -61 & 0.5 & -5 \\
\hline
\end{tabular}


Table II (continued).

\begin{tabular}{ccccc}
\hline \hline $\begin{array}{c}\text { GPS } \\
\text { benchmarck } \\
\text { number }\end{array}$ & $\begin{array}{c}\text { GPS } \\
\text { displacement } \\
(\mathrm{mm})\end{array}$ & $\begin{array}{c}\text { DiffSAR } \\
\text { displacement } \\
(\mathrm{mm})\end{array}$ & Coherence & $\begin{array}{c}\text { GPS-SAR } \\
\text { difference } \\
(\mathrm{mm})\end{array}$ \\
\hline 36 & -56 & -38 & 0.2 & -18 \\
37 & -37 & -15 & 0.2 & -22 \\
38 & -24 & -56 & 0.2 & 32 \\
39 & -17 & -9 & 0.2 & -8 \\
40 & -25 & -25 & 0.4 & 0 \\
41 & -15 & -21 & 0.2 & 6 \\
42 & -9 & -26 & 0.3 & 17 \\
\hline
\end{tabular}

of slopes subject to different velocities and vertical displacements was delineated, modifying previous knowledge (Berardino et al., 2003). Within the valley a progressive and almost linear displacement over time was confirmed. Displacement maps in figs. 3 and 5 detect areas subjected to movements, and hence the active landslides. This information can be used to set the morphodynamic index.

\section{Conclusions}

Results of research on DiffSAR application to the monitoring of Western Basilicata (Maratea and Noce Valley) show the possibility to use algorithms and operative conditions (integrated use of reference GPS networks) such that slow and progressive soil deformations can be detected, even in small areas and in medium-low coherence areas. However, the obtained final maps have some limitations: they do not cover the entire monitored area (in the considered case-study for about $40 \%$ of the territory the coherence coefficient was below 0.3 ) and show some visual artefacts due to the sensor viewing geometry. Obtained data, however, can be integrated by ground based observations and by other remote sensing images. These results encourage applications aimed at studying landslide movements and erosion and deposition processes close to rivers. Therefore, the outlined proce- dures are suitable to develop a project aimed at monitoring permanent changes produced by hydrogeological events and at devising criteria for the definition of a relative damage scale.

\section{REFERENCES}

Achache, J., B. Fruneau and C. Delacourt (1995). Applicability of SAR interferometry for operational monitoring of landslides, in Proceedings of the 2nd ERS Applications Workshop, London, 165-168.

Berardino, P., M. Costantini, G. Franceschetti, A. Iodice, L. Pietranera and V. Rizzo (2002): Differential SAR interferometry for the study of slope instability at Maratea, Italy, in Proceedings of the International Geoscience and Remote Sensing Symposium, Toronto, Canada, 2693-2695.

Berardino, P., M. Costantini, G. Franceschetti, A. Iodice, L. PIETRANERA and V. Rizzo (2003): Use of differential SAR interferometry in monitoring and modelling large slope instability at Maratea (Basilicata, Italy), Eng. Geol., 68, 31-51.

Carnec, C., D. Massonnet and C. King (1996): Two examples of the use of SAR interferometry on displacement-fields of small spatial extent, Geophys. Res. Lett., 23 (24), 3579-3582.

Ferretti, A., C. Prati and F. RocCA (2001): Permanent scatterers in SAR interferometry, IEEE Trans. Geosci. Remote Sensing, 39 (1), 8-20.

FRANCESCHETTI, G. and R. LANARI (1999): Synthetic Aperture Radar Processing (CRC Press, Boca Raton, FL), 25-28.

Goldstein, R.M., H.A. ZEBKer, C.L. WeRner, P.A. Rosen and A. GABRIEL (1994): On the derivation of coseismic displacement field using differential radar interferometry: the Landers earthquake, J. Geophys. Res., 99, 19618-19634. 